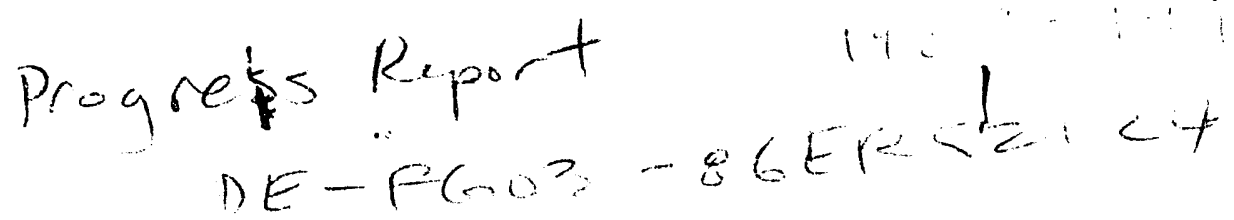

A Research Proposal to

INE IER $|\leq 2| 24-T \mid$

U.S. DEPARTMENT OF ENERGY

FUSION NEUTRONICS EXPERIMENTS AND ANALYSIS

Submitted by

THE REGENTS OF THE UNIVERSITY OF CALIFORNIA

UNIVERSITY OF CALIFORNIA, LOS ANGELES SCHOOL OF ENGINEERING AND APPLIED SCIENCE

LOS ANGELES, CALIFORNIA 90024

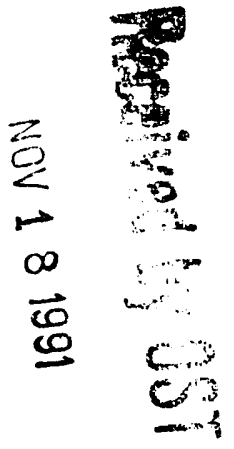

PRINCIPAL INVESTIGATOR:

$\mathrm{DOE} / \mathrm{ER} / 52124--\mathrm{Tl}$

DE92 003399

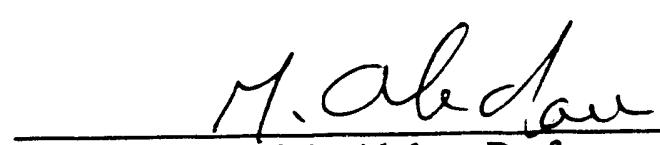

Mohamed A. Abdou, Professor

Mechanical, Aerospace and Nuclear Engineering Department

Office Phone: (213)206-05C1

Desired Starting Date: 11/01/91

Duration: Three years

Estimated Cost: $\$ 2,026,156$

ENDORSEMENTS:
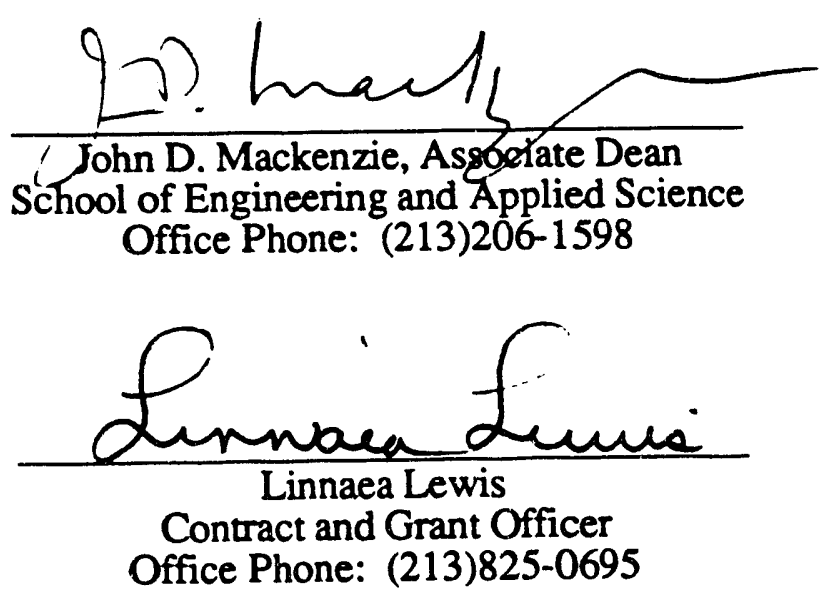

JUNE 1991

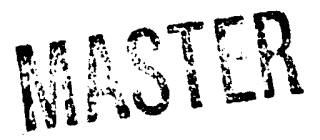

DISTFIBUTION OF THIS DOCUMENT IS UNLIMITEE 


\section{FUSION NEUTRONICS EXPERIMENTS AND ANALYSIS}

\section{INTRODUCTION}

For the past several years, the UCLA program led the neutronics activities in the US. The key part of these activities has been carried out as part of the US-JAERI collaborative program on fusion neutronics. This program of experiments and analysis has made substantial contributions to establishing the foundations of fusion neutronics capabilities that are crucial to the design, construction and operation of near-term fusion devices such as ITER and BPX.

During the past two years, the technical advances made represent a "quantum jump" in the state-of-the-art of fusion neutronics. Some of these advances include: 1) the use of a "point" neutron source to realize a "line" source, which makes the simulation of blanket, shield and plasma facing components for tokamaks much more realistic, 2) the development and successful utilization of a new technique for integral measurements of induced radioactivity and decay heat in fusion materials, 3) the development of a new experimental technique to measure nuclear heating, and 4) substantial reductions in the uncertainties of predicting tritium production rates.

These achievements are "first-of-a-kind" in the history of fusion nuclear R\&D. They provide confidence that we can now proceed with "fusion-specific", particularly "ITERspecific", neutronics R\&D. The comparison of the experimental and analytical results indicate serious issues in some parts of the nuclear data base and calculational methods. Resolving these issues require an aggressive, well focused program of experiments and analysis.

This document is a research proposal for a three year period, FY92, FY93 and FY94. The proposed research involves experiments and analysis to address the most critical neutronics and shielding issues for ITER. Section 2 summarizes progress made during the past year. Section 3 lists some of the key publications from this research activity. Sections 4 and 5 highlight the proposed research activities for FY92 and for FY93 and FY94, respectively. The last section presents the requested budget.

\section{RECENT PROGRESS}

Record accomplishments characterize the experimental and analytical efforts of the past year. Collaboration with JAERI continued on both experiments at FNS and analyses.

During Phase IIIA, bare line source experiments were done to characterize it axially (along the source) and radially (perpendicular to the source). Both neutron energy spectra and dosimetric foil activation rates were measured towards this end. Annular blanket experiment was carried out using assembly built-up radially of $1.5 \mathrm{~cm}$ thick stainless steel, $20 \mathrm{~cm}$ thick clad $\mathrm{Li}_{2} \mathrm{O}, 20 \mathrm{~cm}$ thick $\mathrm{Li}_{2} \mathrm{CO}_{3}$ and $1.6 \mathrm{~cm}$ thick outermost shell of polyethylene. The inner annular region contains air and measures $21.3 \mathrm{~cm} \times 21.3 \mathrm{~cm}$ and extends over a length of $204 \mathrm{~cm}$ as does the surrounding blanket. The line source is simulated by axially moving the annular blanket/detectors at an average speed of $6.1 \mathrm{~mm} / \mathrm{s}$ 
over a length of $200 \mathrm{~cm}$. The experimental measurements of tritium breeding, neutron and gamma energy spectra, gamma heating and dosimetry reactions were carried out along the axis (near inner lining of stainless steel) and in the radial drawers. In Phase IIIB, similar measurements were carried out.

In addition, induced decay $\gamma$ radioactivity measurements were carried out on a number of foil packets that consisted of $\mathrm{Fe}, \mathrm{Ni}, \mathrm{Mo}, \mathrm{Cr}, \mathrm{SS} 316, \mathrm{~W}, \mathrm{Ta}, \mathrm{Zr}, \mathrm{Al}, \mathrm{Sn}, \mathrm{Ag}, \mathrm{Pb}, \mathrm{Zn}, \mathrm{Nb}$, $\mathrm{Ti}, \mathrm{V}, \mathrm{MnCu}$ alloy, $\mathrm{Co}, \mathrm{Mg}, \mathrm{Si}$ and In. The half lives ranged from 10 minutes to 5 years. In addition, nuclear heat deposition rates were measured using microcalorimetric technique near rotating target neutron source which is a point source. The average intensity of $2.5 \times 10^{12} \mathrm{n} / \mathrm{s}$ was realized during the experiments. The employed microcalorimeters consisted of single probes of $\mathrm{Al}, \mathrm{C}, \mathrm{W}, \mathrm{Cu}$ and $\mathrm{Fe}$ and multiple-probe host media of iron and graphite. During Phase IIIB, additional single probes of W, C, Ti, SS-304, Ni and $\mathrm{Mo}$, and multiple-probe host medium of $\mathrm{Cu}$ were employed for nuclear heating measurements.

\subsection{Tritium Breeding Measurements and Analysis}

Tritium breeding measurements were carried out both in Phase IIIA and Phase IIIB. Online lithium glass scintillation, and NE213 liquid scintillation techniques were employed along with the off-line technique employing $\mathrm{Li}_{2} \mathrm{O}$ pellet. These measurements were supplemented by usual complement of dosimetric foil activation, NE213 neutron spectrometry and proton recoil counter spectroscopy.

The analysis for these line source driven experiments needed additional effort as against the previous work related to the point source. Two dimensional DOT5.1 deterministic calculations were preceded by a RUFF calculation with discrete point sources distributed on a $200 \mathrm{~cm}$ long line. Preanalysis showed that 50 points distributed according to gausslegendre quadrature yield better than $0.1 \%$ agreement on axial/radial profiles of tritium breeding/reaction rates in an entire blanket assembly. Each point source is characterized by an energy and angular distribution pre-computed with 3D monte carlo MCNP code. In addition, direct calculations are done with MCNP for a spatially continuous source neutron distribution. FNS (JAERI) did their own calculations using DOT3.5 (deterministic) and MORSE-DD (monte carlo) and JENDL-3. Figures 1 and 2 display ratio, C/E, of computed to experimentally measured values of $T_{7}$, tritium production from ${ }^{7} \mathrm{Li}$, and $\mathrm{T}_{6}$, tritium production from ${ }^{6} \mathrm{Li}$, as a function of radial distance from the central axis, for $\mathrm{Phase}$ IIIA. Mean C/E for $\mathrm{T}_{6}$ is seen to be 1.0 with a standard deviation of $10 \%$. Mean $\mathrm{C} / \mathrm{E}$ for $T_{7}$ is seen to be 0.95 with a standard deviation of $10 \%$. Analysis for Phase IIIB indicates a shift in these trends. Figures 3 to 4 show $C / E$ plots for $T_{7}$ (NE213), and $T_{6}$ (Li glass) for the two phases. Only DOT5.1 calculations have been utilized here. C/E's show decline for $\mathrm{T}_{7}$ and $\mathrm{T}_{6}$ ( $\mathrm{Li}$ glass). However, $\mathrm{C} / \mathrm{E}$ 's for $\mathrm{T}_{6}\left(\mathrm{Li}_{2} \mathrm{O}\right.$ pellet) have been observed to display a reverse trend.

During Phases I through IIIB, it has been observed that $C / E$ 's for $T_{6}$ and $T_{7}$ display different degrees of deviation from unity. The accumulation of extensive data over the years has now led us to have a fresh look at it with a view to decel any possible trends. This, in fact, is even highly desirable as cross-section data has been revised from time to time during this period, on one hand, and the experiments have covered a wide gamut of prototypical assemblies, on the other. A convenient term in this regard is prediction uncertainty and is derived as (C/E-1). This quantity can be used to obtain mean prediction uncertainty, standard deviation on it as well as its smallest and the largest magnitude for each experimental assembly. Figures 5 and 6 show prediction uncertainty plots for $T_{6}$ and 
$\mathrm{T}_{7}$ for Phases I through IIIA. Only DOT5.1 and MCNP calculations have been considered. Blackened box in each horizontal rectangle represents on standard deviation; mean prediction accuracy is represented at center of this box; the rectangle is envelope of all point-wise deviations observed for a given assembly. The abbreviations for different assemblies are listed in Table 1. Broadly, it is to be observed that prediction uncertainty has tended to decline even as the experimental complexity has continued to grow due to increasing prototypicality from Phase I through IIIA.

\subsection{Induced Radioactivity Measurements and Analysis}

The experimental measurements of induced radioactivity were carried out in Phases IIC though IIIB. The irradiated samples have included: $\mathrm{Fe}, \mathrm{Ni}, \mathrm{Cr}, \mathrm{MnCu}$ alloy, $\mathrm{Ti}, \mathrm{Mo}, \mathrm{Zr}$, $\mathrm{Ta}, \mathrm{W}, \mathrm{Si}, \mathrm{Mg}, \mathrm{Al}, \mathrm{V}, \mathrm{Nb}, \mathrm{SS} 316, \mathrm{YBa}_{2} \mathrm{Cu}_{3} \mathrm{O}_{7}, \mathrm{ErBa}_{2} \mathrm{Cu}_{3} \mathrm{O}_{7}, \mathrm{Zn}, \mathrm{Sn}, \mathrm{Ag}, \mathrm{Pb}, \mathrm{Co}$, In and $\mathrm{Au} . \gamma$ spectroscopy of the irradiated samples was done using intrinsic germanium detectors for cooling times ranging from few minutes to a month. Four leading decay radioactivity codes, DKRICF, REAC-2, RACC and THIDA-2 have been used to analyze the measured data. The integrated decay $\gamma$-emission rates (over $100 \mathrm{KeV}$ to $3 \mathrm{MeV}$ of $\gamma$ energy) have been compared in addition to decay $\gamma$ emission spectra. Figures 7 a through 7c show C/E's for different materials as calculated from DKRICF, REAC-2 and THIDA-2 codes. Figures 8 through 10 show C/E's for individual materials. It is observed that: (i) generally, much better agreement is found between computed (C) and experimentally measured $(E)$ values for integrated $\gamma$ emission rates as against the detailed $\gamma$ spectra, (ii) $C / E$ ratios for integrated $\gamma$ emission rates are found to range from 0.001 to 300 , though most of the ratios cluster between 1 to 2 . Significant discrepancies are obtained on $C / E$ ratios for a number of cases for the four codes used above. Most of the observed discrepancies are due to (a) missing or fundamental decay $\gamma$-ray data, e.g., (1) missing decay data in DKRICF for ${ }^{186} \mathrm{Ta},{ }^{187} \mathrm{~W},{ }^{181} \mathrm{~W},{ }^{90 \mathrm{~m} Y},{ }^{86} \mathrm{Rb},{ }^{88} \mathrm{Y}$, etc., (2) wrong decay $\gamma$-intensities for W products in THIDA-2, (b) inaccurate activation cross sections, e.g., for $\mathrm{Zr}, \mathrm{Mo}$, W, in DKRICF, REAC-2, RACC, (c) errors on computed neutron energy spectra- especially low energy component in relation to $(n, \gamma)$ products like ${ }^{56} \mathrm{Mn}$ from $\mathrm{Mn}$, (d) various experiment related factors, essentially poor counting statistics for weak neutron induced reactions.

It is instructive to look at tungsten related decay $\gamma$ radioactivity data to feel how we have arrived at deceling discrepancies in the decay and cross-section data of different code systems. Measurement and analysis of decay radioactivity data on tungsten has been discussed in length in our memo sent in March 1991 to international community. Figures 11 and 12 show $\mathrm{C} / \mathrm{E}$ for integrated decay $\gamma$ emission and reaction rates respectively. All four code systems were found deficient in predicting the measured data. Decay data was discrepant in all four code systems: half lives of both ${ }^{184} \mathrm{Ta}$ and ${ }^{186} \mathrm{Ta}$ were entered wrongly in RACC and DKRICF; ${ }^{187} \mathrm{~W}$ decay $\gamma$ emission data is erroneously assigned to ${ }^{186} \mathrm{~W}$, a stable nucleus, in RACC; decay $\gamma$ yields for ${ }^{179} \mathrm{~mW}\left(t_{1 / 2}=6.4 \mathrm{~m}\right)$ were up by four orders in REAC-2, whereas, the RACC and DKRICF din not have any data on this isotope; $\mathrm{i} 87 \mathrm{~W} \gamma$-yields were included as $83.2 \%$ against $0.35 \%$ in $200-400 \mathrm{KeV}$ range, and as $82.6 \%$ in $100-200 \mathrm{KeV}$ range as against $8.7 \%$. Even reaction-rate-wise C/E's showed a large scatter for all important products. $\mathrm{C} / \mathrm{E}$ for ${ }^{187} \mathrm{~W}$ ranged from 1.5 (THIDA-2) to 5 (DKRICF). For all major products, C/E's from THIDA-2 were closest to 1. The comparison of neutron energy wise cross sections with those from ENDF/B-VI showed a 
stark need to improve the cross-section data in all the code systems and, practically, for all major reactions. Ad hoc decay data and cross-section improvements have led to better agreement between the measurements and the computations for tungsten (THIDA-2, RACC, DKRICF, REAC-2), zirconium and some other materials. This points to a crying need to launch a systematic effort to bring out a highly recommended overhaul of the decay data and cross-section libraries of these four code systems.

\subsection{Nuclear Heating Measurements and Analysis}

Direct nuclear heat deposition rate measurements have been carried out during Phases IIC through IIIB. Microcalorimetric technique has been used with single and multiple probes. Point neutron source of rotating neutron target (RNT) was needed due to requirement of higher neutron intensity for this technique. Figure 13 shows the experimental geometry and Figure 14 explains the underlying principle of IEEE488 bus based electronic instrumentation employed for online data acquisi ion. It needs to be pointed out that the major objectives of this activity have been (i) to establish the experimental methodology for direct nuclear heating measurements using microcalorimetry technique (with thermistors and platinum RTD's as thermal sensors), (ii) to accumulate nuclear heating rate data base for integral verification of kerma factors/heating numbers. The first measurements were carried out during June 1989; the subsequent measurements have been conducted during December 1989 and November 1990. Small probes of materials have been irradiated in close vicinity of rotating target. A typical probe contained a core measuring $2 \mathrm{~cm}$ diameter by $2 \mathrm{~cm}$ length. Probes of leading candidates for different applications have been investigated: molybdenum, tungsten, titanium, graphite (plasma facing components), copper (magnet coils), iron, stainless steel 304, nickel (structural material components) and aluminum. Figures 15 and 16 show typical nuclear heat deposition profiles in iron and tungsten single probes respectively. The measured temperature-change rates have ranged from $30 \mu \mathrm{K} / \mathrm{s}$ (iron) to $330 \mu \mathrm{K} / \mathrm{s}$ (graphite). The corresponding nuclear heating rates have ranged from $\sim 35 \mu \mathrm{W} / \mathrm{g}$ (tungsten) to $-225 \mu \mathrm{W} / \mathrm{g}$ (graphite). The average target-probe distance in these experiments has ranged from 5 to $8 \mathrm{~cm}$. Signal to noise $(\mathrm{S} / \mathrm{N})$ ratio improves for shorter distances. The closeness of a probe to the target is susceptible to add to experimental error, due to uncertainty in relative positioning of the probe in the vacuum chamber, even though great care was taken to minimize this uncertainty.

The measurements have been analyzed using both ENDF/B-V and JENDL-3 nuclear data. The codes that have been used include: MCNP with ENDF/B-V, DOT3.5 with JENDL-3. Many kerma factor/heating number libraries were used in MCNP computations. It was found that there is a large divergence in the heating rates computed by different kerma factor libraries for same material, for example, there was $40 \%$ deviation around the mean for aluminum. $C / E$ value with $E N D F / B-V$ data varied from 0.4 to $1.8 ; C / E$ varied from 1.3 to 1.9 with JENDL-3 data. Table 2 brings out the flavour of discrepancies observed with different data bases available with MCNP.

Experimental measurements have also been made in three host media of graphite, iron and copper that housed small probes of host medium, tungsten and graphite. Each host medium measured $85 \mathrm{~mm}$ diameter $\times 100 \mathrm{~mm}$ length. A typical probe was $10 \mathrm{~mm}$ long with a diameter of $1 \mathrm{~mm}$ and was located in the host medium such that there was no physical contact between the two. The analysis has revealed large deviations in $\mathrm{C} / \mathrm{E}$, for example, for iron host, C/E varies from 0.5 to 1.1 with ENDF/B-V data. To conclude, it is necessary to continue perfecting the experimental technique, on one hand, and intercompare various kerma factor libraries, on the other, so as to understand, and, possibly, remove the underlying sources of discrepancy in $C / E$ 's. 


\section{PUBLICATIONS}

(A) Published Articles

(1) M.Z. Youssef, Y. Watanabe, A. Kumar, Y. Oyama, and K. Kosako, "Analysis for the Simulation of Line Source By a $14 \mathrm{MeV}$ Moving Point Source and Impact on Blanket Characteristics: The USDOE/JAERI Collaborative Program on Fusion Neutronics," Fusion Technology, 19 (1991)1843-1852.

(2) A. Kumar, M.Z. Youssef, Y. Ikeda, C. Konno, and Y. Oyama, "Experiments and Analysis for Measurements of Decay Heat Related Induced Activities in Simulated Line Source Driven D-T Neutron Fields of Phase IIIA: USDOE/JAERI Collaborative Program on Fusion Neutronics," Fusion Technology, 19 (1991) 1859-1866.

(3) T. Nakamura, Y. Oyama, Y. Ikeda, C. Konno, H. Maekawa, K. Kosako, M.Z. Youssef, and M.A. Abdou, "A Line D-T Neutron Source Facility for Annular Blanket Experiment: Phase III of the JAERI/USDOE Collaborative Program on Fusion Neutronics," Fusion Technology, 19 (1991) 1873-1878.

(4) Y. Oyama, C. Konno, Y. Ikeda, H. Maekawa, K. Kosako, T. Nakamura, A. Kumar, M. Youssef, and M.A. Abdou, and E. Bennett, "Annular Blanket Experiment Using a Line DT Neutron Source: Phase IIIA of the JAERI/USDOE Collaborative Program on Fusion Neutronics," Fusion Technology, 19 (1991) 18791884.

(5) C. Konno, Y. Oyama, Y. Ikeda, K. Kosako, H. Maekawa, T. Nakamura, A. Kumar, M.Z. Youssef, M.A. Abdou, and E.F. Bennett," Measurements of the Source Term for Annular Blanket Experiment with a Line Source: Phase IIIA of JAERI/USDOE Collaborative Program on Fusion Neutronics," Fusion Technology, 19 (1991) 1885-1890.

(6) M. Z. Youssef, A. Kumar, M. Abdou, M. Nakagawa, K. Kosako, Y. Oyama, and T.Nakamura, "Analysis for Heterogeneous Blankets and Comparison to Measurements: Phase IIC Experiments of the USDOE/JAERI Collaborative Program on Fusion Neutronics", Fusion Technology, 19 (1991) 1891-1902.

(7) A. Kumar, M.A. Abdou, Y. Ikeda, and T. Nakamura, "Analysis of Induced Acrivities Measurements Related to Decay Heat in Phase IIC Experimental Assembly: USDOE/JAERI Collaborative Program on Fusion Neutronics Experiments," Fusion Technology, 19 (1991) 1909-1918.

(8) Y. Oyama, S. Yamaguchi, K. Tsuda, C. Konno, Y. Ikeda, H. Maekawa, T. Nakamura, K. Porges, and E. Bennett, "Measured Characteristics of Be Multilayered and Coolant Channel Blankets: Phase IIC Experiments of the JAERI/USDOE Collaborative Program on Fusion Neutronics, "Fusion Technology, 19 (1991) 19551960.

(9) Y. Ikeda, C. Konno, T. Nakamura, A. Kumar, and M.A. Abdou, "Experiment on Induced Activities and Decay-Heat in Simulated D-T Neutron Fields: JAERI/USDOE 
Collaborative Program on Fusion Neutronics, " Fusion Technology, 19 (1991) 1961 1966.

(10) M.Z. Youssef, and Y. Watanabe, "Study on the Accuracy of Several Beryllium Evaluations and Comparison of Measured and Calculated Data on Reaction Rates and Tritium Production Distributions, " Fusion Technology, 19 (1991) 1967-1973.

(11) A. Kumar, Y. Ikeda, and C. Konno, "Experimental Measurements and Analysis of Nuclear Heat Deposition Rates in Simulated D-T Neutron Environment: JAERI/USDOE Collaborative Program on Fusion Neutronics Experiments," Fusion Technology, 19 (1991) 1979-1988.

(12) A. Kumar, M.A. Abdou, Y. Ikeda, and C. Konno, "Radioactivity and Nuclear Heating Measurements for Fusion Applications, " paper to appear in proceedings of 16th Symposium on Fusion Technology, held 3-7 September, 1990, Abingdon, U.K.

(13) A. Kumar, "Measurement and Analysis of Decay Radioactivity Data on Tungsten," UCLA memo dated February 19, 1991, distributed internationally on March 21, 1991.

(B) Articles to Appear

(14) M.A. Abdou, H. Maekawa, and A. Takahashi, "Recent Advances in Experimental Techniques and Calculational Methods for Fusion Neutronics," paper to appear in Fusion Engineering and Design as a part of proceedings of ISFNT 2, held 2-7 June, 1991, Karlsruhe, Germany.

(15) Y. Oyama, C. Konno, Y. Ikeda, H. Maekawa, K. Kosako, T. Nakamura, A. Kumar, M. Youssef, M. Abdou, E. Bennett, "Phase-III Experimental Results of JAERI/USDOE Collaborative Program on Fusion Neutronics, " paper to appear in Fusion Engineering and Design as a part of proceedings of ISFNT 2, held 2-7 June, 1991, Karlsruhe, Germany.

(16) M.Z. Youssef, A. Kumar, M.A. Abdou, Y. Oyama, K. Kosako, T. Nakamura, "Post-analyses for the line source Phase IIIA Experiments of the USDOE/JAERI Collaborative Program on Fusion Neutronics, " paper to appear in Fusion Engineering and Design as a part of proceedings of ISFNT 2, held 2-7 June, 1991, Karlsruhe, Germany.

(17) T. Nakamura, Y. Oyama, Y. Ikeda, H. Maekawa, C. Konno, K. Kosako, F. Maekawa, M.A. Abdou, M.Z. Youssef, A. Kumar, "Overview of the Latest Experiments under the JAERI/USDOE Collaborative Program on Fusion Neutronics," paper to appear in Fusion Engineering and Design as a part of proceedings of ISFNT 2, held 2-7 June, 1991, Karlsruhe, Germany.

(18) Y. Oyama, K. Kosako, M. Nakagawa, and T. Nakamura, "Comparative Study of Systems and Nuclear Data in C/E ratios for a Series of JAERI/USDOE Collaborative Fusion Blanket Experiments, " paper to appear in Fusion Engineering and Design as a part of proceedings of ISFNT 2, held 2-7 June, 1991, Karlsruhe, Germany.

(19) Y. Ikeda, C. Konno, Y. Oyama, T. Nakamura, A. Kumar, M.Z. Youssef, and M.A. Abdou, "Experimental Verification of the Current Data and Methods for Induced Radioactivity and Decay Heat Calculation in D-T Fusion Reactors, " paper to appear 
in Fusion Engineering and Design as a part of proceedings of ISFNT 2, held 2-7 June, 1991, Karlsruhe, Germany.

(20) A. Kumar, Y. Ikeda, C. Konno, K. Kosako, M.Z. Youssef, M.A. Abdou, T. Nakamura, and Y. Oyama, "Direct Nuclear Heating Measurements in Fusion Neutron Environment and Analysis, " paper to appear in Fusion Engineering and Design as a part of proceedings of ISFNT 2, held 2-7 June, 1991, Karlsruhe, Germany.

(21) M.Z. Youssef, A. Kumar, M. Abdou, "The Prediction Capability for Tritium Production and Other Reaction Rates in Various Systems Configurations for a Series of the USDOE/JAERI Collaborative Fusion Blanket Experiments, " paper to appear in Fusion Engineering and Design as a part of proceedings of ISFNT 2, held 2-7 June, 1991, Karlsruhe, Germany.

\title{
DISCLAIMER
}

\begin{abstract}
This report was prepared as an account of work sponsored by an agency of the United States Government. Neither the United States Government nor any agency thereof, nor any of their employees, makes any warranty, express or implied, or assumes any legal liability or responsibility for the accuracy, completeness, or usefulness of any information, apparatus, product, or process disclosed, or represents that its use would not infringe privately owned rights. Reference herein to any specific commercial product, process, or service by trade name, trademark, manufacturer, or otherwise does not necessarily constitute or imply its endorsement, recommendation, or favoring by the United States Government or any agency thereof. The views and opinions of authors expressed herein do not necessarily state or reflect those of the United States Government or any agency thereof.
\end{abstract}



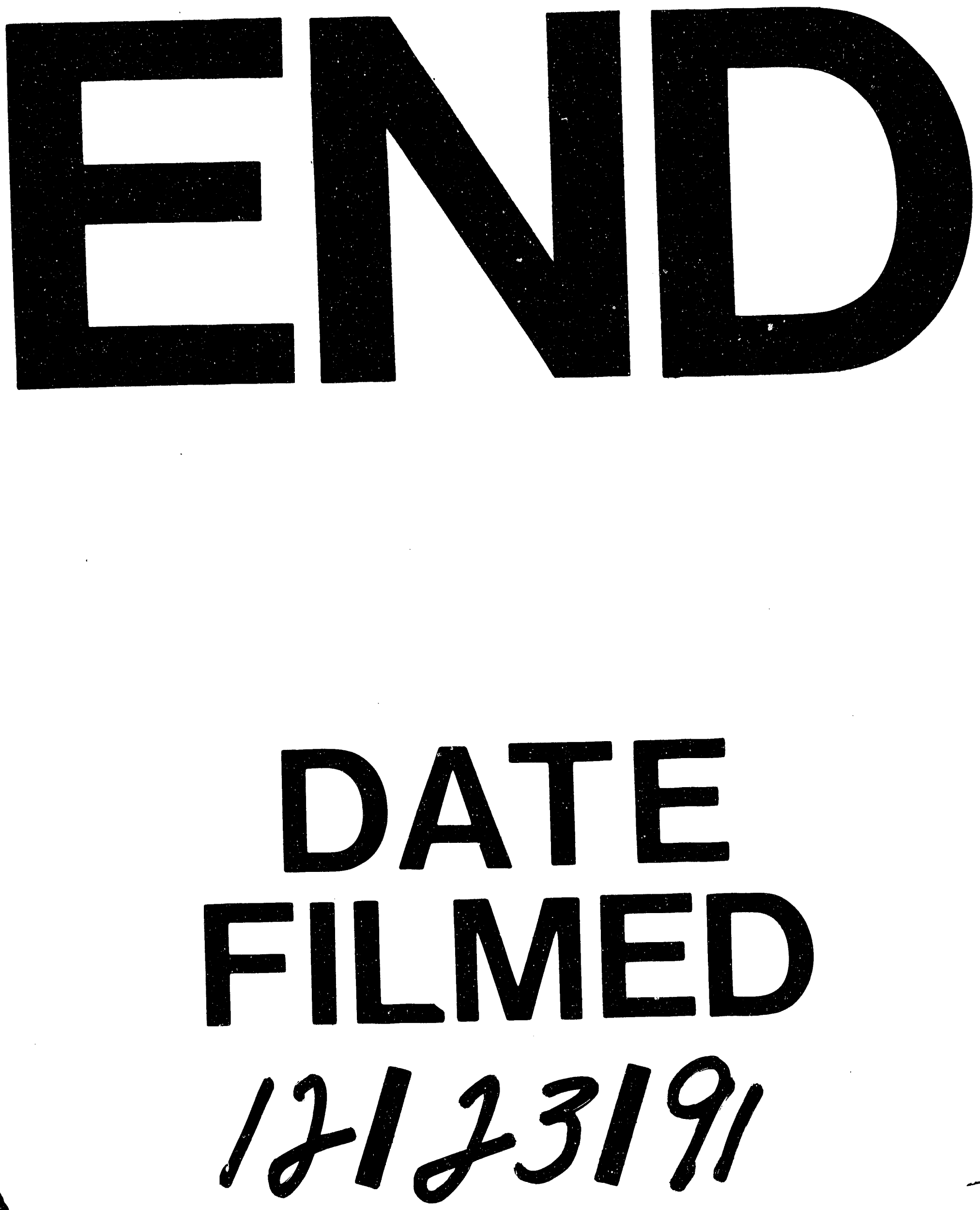
